\title{
Realidad aumentada como técnica didáctica en la enseñanza de temas de cálculo en la educación superior. Estudio de caso
}

\begin{abstract}
Augmented Reality as a Didactic Technique in the Teaching of Calculus Topics in Higher Education. Case Study
\end{abstract}

A realidade aumentada como técnica didática no ensino de disciplinas de cálculo no ensino superior. Estudo de caso

Efrén Berumen López

Tecnológico Nacional de México, Instituto Tecnológico Superior Jerez, México efren.bl@jerez.tecnm.mx https://orcid.org/0000-0003-0224-4300

Salvador Acevedo Sandoval

Tecnológico Nacional de México, Instituto Tecnológico Superior Jerez, México salvador.acevedo@tecjerez.edu.mx https://orcid.org/0000-0003-1871-3589

Susana Reveles Gamboa

Tecnológico Nacional de México, Instituto Tecnológico Superior Jerez, México susana.rg@jerez.tecnm.mx https://orcid.org/0000-0001-8634-9569 


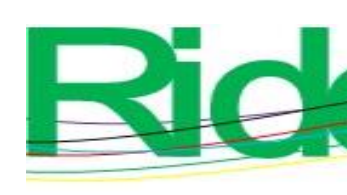

Revista Iberoamericana para la
Investigación y el Desarrollo Educativo
ISSN $2007-7467$

\section{Resumen}

En este documento se presenta un estudio cuasiexperimental para medir el impacto de la realidad aumentada en el proceso de enseñanza-aprendizaje con alumnos del Tecnológico Nacional de México, campus Jerez, de la carrera de Contador Público. Se consideró un grupo de control y un grupo experimental. En el grupo experimental se utilizó una app de realidad aumentada como herramienta de apoyo, mientras que en el grupo de control se realizaron las clases de manera tradicional. En ambos casos la intervención se llevó a cabo exclusivamente en la asignatura de "Cálculo diferencial e integral". Se utilizó la prueba no paramétrica de Mann-Whitney-Wilcoxon para mostrar si había diferencia entre ambos grupos. Los resultados muestran que no hay diferencia si se utiliza realidad aumentada como herramienta de apoyo o se presentan las clases de forma tradicional. Sin embargo, los estudiantes que utilizaron la realidad aumentada consideraron que esta les ayudó a mantener la atención en el transcurso de la asignatura, así como a despertar o aumentar el interés por el contenido impartido.

Palabras clave: educación superior, enseñanza de las matemáticas, realidad aumentada.

\section{Abstract}

In this document, a quasi-experimental study is presented which was designed to measure the impact of augmented reality on the teaching-learning process involving accounting majors from the Jerez Campus of the Mexican higher education institution Tecnológico Nacional de México. A control group and an experimental group were formed with students enrolled in the same Differential and Integral Calculus subject. An augmented reality app was used with the experimental group as a support teaching tool, while with the control group the lessons were carried out in a traditional fashion. The Mann-Whitney-Wilcoxon nonparametric test was used to show if there were any difference in academic performance between the groups. The results show that there is no difference whether augmented reality is used as a support teaching tool or classes are taught in a traditional fashion. However, the students who used augmented reality considered that it helped them to keep focused throughout the course, and that it likewise awakened or increased their interest on the subject.

Keywords: higher education, mathematics teaching, augmented reality. 


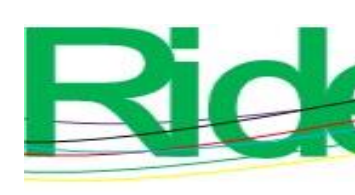

Revista Iberoamericana para la Investigación y el Desarrollo Educativo ISSN $2007-7467$

\section{Resumo}

Este documento apresenta um estudo quase experimental para medir o impacto da realidade aumentada no processo de ensino-aprendizagem com alunos do Instituto Tecnológico Nacional do México, campus Jerez, da carreira de Contador Público. Um grupo controle e um grupo experimental foram considerados. No grupo experimental, um aplicativo de realidade aumentada foi utilizado como ferramenta de apoio, enquanto no grupo controle as aulas foram ministradas de forma tradicional. Em ambos os casos a intervenção foi realizada exclusivamente na disciplina de "Cálculo diferencial e integral". O teste não paramétrico de Mann-Whitney-Wilcoxon foi usado para mostrar se havia diferença entre os dois grupos. Os resultados mostram que não há diferença se a realidade aumentada é utilizada como ferramenta de apoio ou as aulas são apresentadas de forma tradicional. No entanto, os alunos que utilizaram a realidade aumentada consideraram que ela os ajudava a manter a atenção ao longo do curso, bem como a despertar ou aumentar o interesse pelos conteúdos ministrados.

Palavras-chave: ensino superior, ensino de matemática, realidade aumentada.

Fecha Recepción: Septiembre 2020

Fecha Aceptación: Marzo 2021

\section{Introducción}

El ser humano vive actualmente en un estado cambiante y versátil. Las nuevas formas de comunicación han revolucionado la manera en que se crea y difunde el conocimiento, la manera en que se dan las relaciones interpersonales y el modo en que se imparte la educación. Esto en gran medida se debe a la invención del Internet. Los últimos 100 años han contribuido al desarrollo de estos cambios sociales (De la Horra, 2017). Las tecnologías de la información y comunicación (TIC) han influido en prácticamente todas las áreas del conocimiento: ciencias de la salud, ciencias sociales, bellas artes, economía, administración, ingeniería, ciencias de la educación, por mencionar algunas.

En el campo de la educación, al igual que en muchos otros, conforme las tecnologías progresan, los procesos para interactuar también lo hacen, de ahí que el ser humano se encuentre inmerso en una dinámica de cambio constante (Ballesteros y Bernal, 2016). El uso de la tecnología dentro de las aulas de clase en cualquier nivel educativo debe ser un apoyo para las dificultades de aprendizaje que el estudiante posea, sin perder de vista que el aprendizaje y el conocimiento a adquirir deben ser el eje sobre el que las actividades 


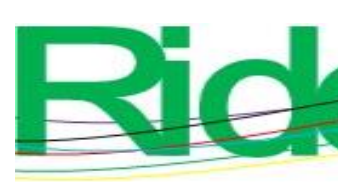

Revista Iberoamericana para la Investigación y el Desarrollo Educativo ISSN 2007 - 7467

educativas giren, y no la tecnología en sí misma (Rubio, Cabero, Leiva, Moreno, Barroso y López, 2016). Por su parte, Cupitra y Duque (2018) mencionan que el objetivo de las TIC en la educación debe ser el de generar e imaginar nuevas estrategias pedagógicas. Johnson, Smith, Willis, Levine y Haywood (2016, citados en Maquilón, Mirete y Avilés, 2017) destacan de entre las tecnologías que se están desarrollando actualmente a la realidad aumentada (RA), la cual está abriendo un espacio como recurso innovador en el terreno educativo.

El concepto de $R A$ se refiere a una tecnología basada en la observación del mundo real aumentada con información adicional generada por computadora (Buitrago, 2015). Berryman (2012, citado en Barba, Yasaca y Manosalvas, 2015) añade que la RA es una tecnología de imágenes en tercera dimensión que impone datos originados por las computadoras en objetos o lugares geográficos en el mundo real. Esta tecnología tiene tres características. La primera es que combina lo real y lo virtual, la segunda peculiaridad es que es interactiva y en tiempo real y la tercera singularidad es que se registra en 3D (Azuma, 1997). El concepto fue introducido por primera vez por el estadounidense Tom Caudell en 1992, investigador de la empresa aeronáutica Boeing, tras desarrollar un sistema experimental que tenía como meta ayudar a los trabajadores con el ensamblado de cables para sus aeronaves (Álvarez, Castillo, Pizarro y Espinoza, 2017). Esto indica que se trata de un término acuñado dentro de la industria y no en el ámbito académico.

Algunas de las problemáticas que comúnmente presentan las instituciones de nivel superior son los altos índices de reprobación y deserción. Particularmente, el bajo rendimiento académico en las asignaturas de matemáticas es uno de los problemas a los que semestralmente se enfrenta el Instituto Tecnológico Superior de Jerez (ITSJ), uno de los campus del Tecnológico Nacional de México (TecNM). Teniendo en cuenta esta situación, se consideró que el diseño y el uso de una aplicación (app) de RA motivaría el aprendizaje de algunos temas selectos de matemáticas, en específico en una de las asignaturas que presenta mayores índices de reprobación, Cálculo.

En primer lugar, se diseñó una app de RA con el tema de funciones. De la Horra (2017) menciona que existen diferentes niveles de RA de acuerdo con el tipo de interactividad: 


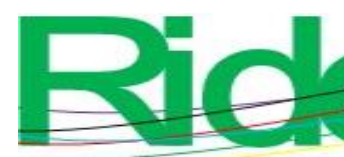

e

- $\quad$ Nivel cero. Códigos QR.

- $\quad$ Nivel uno. RA con marcadores.

- Nivel dos. RA para geolocalización.

- $\quad$ Nivel tres. RA con visores (HDM, por sus siglas en inglés).

El diseño de app se situó en el nivel dos, ya que no necesita marcadores específicos para funcionar, y a pesar de no utilizar completamente la geolocalización para establecer una posición exacta, utiliza capacidades que le permiten al dispositivo móvil saber su ubicación e interactuar con los objetos 3D y el usuario. Esto se debe, según Amadeo (2017), a tres tecnologías clave: 1) seis grados de libertad permiten que el teléfono comprenda y rastree su posición en relación con el mundo, utilizando un proceso llamado localización y mapeo simultáneos (SLAM, por sus siglas en inglés); 2) la comprensión ambiental permite que el teléfono detecte el tamaño y la ubicación de superficies planas horizontales como el suelo o una mesa, y 3) la estimación de luz permite al teléfono valorar las condiciones de iluminación actuales del entorno.

En segundo lugar, se realizó un diseño cuasiexperimental con un grupo de control y otro experimental. En el grupo de control se llevaron las clases de manera tradicional y en el grupo experimental se realizaron actividades utilizando la app de RA durante la enseñanza de funciones. Al final del tema se aplicó una evaluación postest en ambos grupos para medir su rendimiento académico.

Llegado a este punto no está de más especificar que la pregunta que guio el proceso de investigación fue la siguiente: ¿el uso de la RA permitirá mejorar el rendimiento académico de los alumnos en el tema de funciones de la asignatura de Cálculo?

Para el diseño de la herramienta didáctica se consideraron dos elementos principales. El primer elemento fue que la RA es una herramienta didáctica que plantea una dinámica activa (Maquilón et al., 2017). Asimismo, Pedraza, Amado, Lasso y Munévar (2017) mencionan que la RA cuenta con un enfoque constructivista, ya que incluye al estudiante dentro de un contexto físico y social en el mundo real. El segundo aspecto tiene que ver con el hecho de que la herramienta fue diseñada para tratar el tema de funciones debido a que este proporciona fundamentos para tópicos subsecuentes de cálculo, especialmente para el de límites. El concepto de límites es fundamental para muchos problemas de la ingeniería y física, entre otras ramas; de hecho, el cálculo es definido como el estudio de los límites (Purcell, Varberg y Rigdon, 2007). 


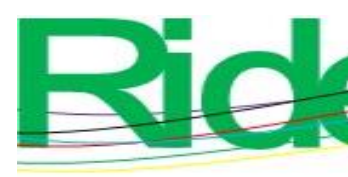

Revista Iberoamericana para la
Investigación y el Desarrollo Educativo
ISSN $2007-7467$

A partir de la segunda mitad del siglo XX, el aumento de las instituciones de nivel superior en América Latina ha sido considerable. Sin duda la educación superior juega un rol importante en el desarrollo de la sociedad. Sin embargo, dicho aumento trae consigo dificultades propias, tales como el bajo rendimiento de los estudiantes y los altos índices de reprobación. En el caso de nuestro país, México, los indicadores de rezago, deserción escolar y eficiencia terminal son algunas de las principales variables que explican los niveles de rendimiento académico deficientes (Edel, 2003).

Por ello es necesario innovar las prácticas educativas existentes, lo que contribuirá en cierta medida a mejorar el rendimiento académico de los estudiantes. El uso de las TIC es una herramienta profesional que todo docente debe emplear. En esa línea, Cabero y Barroso (2016) mencionan que si un docente desea adentrarse al uso de las TIC requiere de apoderarse del conocimiento de tres áreas: tecnología, contenido y conocimiento pedagógico. Particularmente, la RA es una herramienta emergente e innovadora que mejora el aprendizaje de los contenidos (Del Cerro y Morales, 2017).

Tejedor y Muñoz (2007) mencionan que en el nivel superior la mayor parte de la deserción escolar se presenta en los primeros años de carrera, especialmente en el primer y segundo año. Sin perder de vista este punto, la presente investigación se desarrolló con alumnos de primer año. Además, Ocampo, Martínez, De las Fuentes y Zataraín (2010) señalan que en el nivel de enseñanza superior los mayores índices de reprobación se presentan en matemáticas: por ese motivo la interverción se llevará en la asignatura de Cálculo.

Existen varias referencias importantes de investigaciones relacionadas con el uso de las TIC en la educación superior, particularmente en la RA.

En primer lugar, se analizaron artículos relacionados con la revisión sistemática del uso de la RA. Saltan y Arslan (2017), por ejemplo, filtraron, como parte de su revisión sistemática, 23 artículos de una búsqueda de 102. Los hallazgos de esta investigación evidencian un mejor desempeño académico, aumento en el compromiso y satisfacción de los estudiantes en los entornos educativos favorecidos con el uso de la RA. Igualmente, Lopes, Sartor, Pozzebon y Ferenhof (2019) realizaron una revisión sistemática para verificar de qué forma la RA viene siendo utilizada en la educación. Entre los resultados encontrados se menciona que la utilización de la RA en la educación en los alumnos aumentó la motivación y el desempeño académico. Lorenzo y Scagliarini (2018), por su parte, realizaron una 


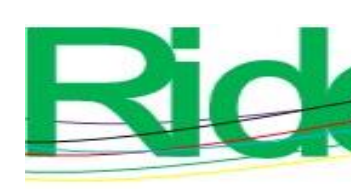

Revista Iberoamericana para la Investigación y el Desarrollo Educativo ISSN 2007 - 7467

investigación sobre el estado de producción científica sobre la RA en la educación. Revisaron 347 artículos publicados en el periodo 1990-2018 y encontraron que entre el 2015 y el 2017 se ha producido la mayor cantidad de artículos relacionados con la RA en la educación (71\%), esto es, la RA, en el ámbito educativo, es un tema que tiene aún mucho por aportar, ya que es una tecnología que sigue en auge.

En segundo lugar, se analizaron artículos que desarrollan experiencias de RA en el proceso de enseñanza-aprendizaje en la educación superior. Tal y como el de Barba et al. (2015), quienes realizaron una valoración global y específica del impacto de la RA sobre el proceso de enseñanza-aprendizaje en 63 estudiantes de Anatomía III en la Escuela Superior Politécnica de Chimborazo del Ecuador. A partir de un diseño cuasiexperimental con un grupo de control y otro experimental, sus resultados muestran que la RA potencia escenarios formativos más motivadores, colaborativos e interactivos y ayuda a una educación más abierta y creativa. Mientras que Cabero, Fernández y Marín (2017) emprendieron un estudio con 148 estudiantes en la Universidad de Sevilla, España, y encontraron que hay una relación significativa entre la motivación y la utilización de apuntes con objetos de RA. Por otro lado, Redondo, Fonseca, Sánchez y Navarro (2017) describen una experiencia educativa con 25 estudiantes de arquitectura usando RA en dispositivos móviles para el aprendizaje de conceptos de diseño urbano. Al igual que en el caso anterior, a partir de un diseño cuasiexperimental con un grupo de control y otro experimental, encontraron que hubo una mejora significativa en las calificaciones del grupo experimental. Al final, los datos obtenidos apoyaron la hipótesis de que la tecnología de RA es una herramienta valiosa en el campo de la arquitectura.

En otras áreas del conocimiento tales como las matemáticas, la física y la química también se encontraron algunas experiencias con el uso de estrategias de RA. Buitrago (2015) realizó una investigación con 83 estudiantes de ingeniería Industrial de la Escuela Colombiana de Carreras Industriales; analizó la asociación entre el logro de aprendizajes en matemáticas, el estilo cognitivo y la RA; elaboró un diseño cuasiexperimental con un grupo de control y otro experimental. Los resultados muestran, en suma, que es significativo desarrollar ambientes virtuales de aprendizaje. Asimismo, el estudio de Álvarez et al. (2017), quienes desarrollaron una experiencia de RA en el proceso de enseñanza-aprendizaje del tópico de mecánica de fluidos, concluye que la RA permite mejores resultados en cuanto a la adquisición de conocimientos. De igual manera, el trabajo de Parroquín, Ramírez, González 


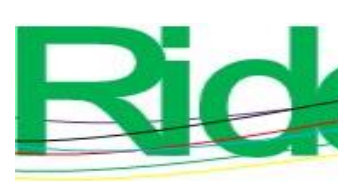

Revista Iberoamericana para la
Investigación y el Desarrollo Educativo
ISSN $2007-7467$

y Mendoza (2013) describe el desarrollo de una aplicación de software que usa RA para le enseñanza de la física con un grupo experimental (con el uso del software) y un grupo de control (sin el uso del software) y comprobaron que el primero tiene una ganancia sobre el segundo. Finalmente, el estudio realizado por Martínez, García y Escalona (2017) creó objetos de RA y fueron aplicados como medios de enseñanza en el estudio de compuestos químicos. Los resultados mostraron que la RA contribuyó a aumentar el interés por las asignaturas y ampliar la comprensión de los contenidos.

Si bien es cierto que existe una variedad de estudios que reportan el uso de la RA en la educación, el experimento realizado en esta investigación, como caso de estudio, se dirigió al uso de esta tecnología en el área de las matemáticas, particularmente en el tema de funciones. Esta asignatura, por su naturaleza de abstracción, es compleja de enseñar y difícil de comprender/aprender. Parte de la importancia del estudio radica en la creación e implementación de la app de RA al interior de la institución. La app fue generada con base en el programa de estudio de la materia de Cálculo del TecNM.

\section{Materiales y métodos}

\section{Diseño de la investigación}

Se diseñó una investigación cuasiexperimental. Así, considerando que es un estudio de caso, se tomó como muestra al segundo semestre de la carrera de Contador Público (CP) del ITSJ del semestre enero-junio 2020, conformado por 21 estudiantes. Este se dividió en dos: un grupo de control (10 estudiantes) y un grupo experimental (11 estudiantes). En ambos grupos (control y experimental) se impartió el tema de funciones de la asignatura de "Cálculo diferencial e integral". Es importante mencionar que previo al experimento se les pidió permiso y consentimiento a los estudiantes para realizar dicho experimento. En las clases del grupo experimental se trabajó con una app de RA para dispositivos móviles que previamente se diseñó y desarrolló específicamente para este fin, debido a que no existía una aplicación con las características requeridas (en el siguiente apartado se expone con más detenimiento este punto). Mientras que en el grupo de control se llevaron las clases de manera tradicional. No hubo mediciones a priori, pero sí a posteriori por medio de una serie de actividades relacionadas con el tema de funciones. El proceso se realizó de la siguiente manera: 


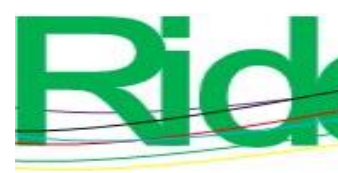

Revista Iberoamericana para la
Investigación y el Desarrollo Educativo
ISSN $2007-7467$

- $\quad$ Se diseñó y desarrolló una app de RA centrada en el tópico de funciones.

- $\quad$ Se eligió un grupo experimental del ITSJ (11 estudiantes).

- $\quad$ Se eligió un grupo de control del ITSJ (10 estudiantes).

- $\quad$ En el grupo experimental se utilizó la app de RA como herramienta de apoyo en el tema de funciones.

- $\quad$ En el grupo de control se realizaron las clases de manera tradicional en el tema de funciones.

- Se aplicó una evaluación postest a ambos grupos para conocer el impacto educativo en cada uno de ellos.

- Se aplicó una encuesta tipo Likert de 11 ítems (anexo 1) al grupo experimental con el fin de recoger información de los estudiantes respecto a su conocimiento de la RA, la disponibilidad de recursos para su aplicación, criterios motivacionales y criterios sobre el uso de la RA, tomada de Martínez et al. (2017).

Para comparar si existía o no diferencia entre las calificaciones de la evaluación postest entre ambos grupos (control y experimental), se aplicó la prueba no paramétrica de Mann-Whitney-Wilcoxon, también conocida como prueba de Mann-Whitney o como prueba de la suma de los rangos de Wilcoxon (Anderson, Sweeney y Williams, 2008). La prueba se utilizó para el caso de muestras grandes $(n \geq 10)$. Además, dicha prueba no requiere que los datos estén distribuidos normalmente y el requisito es que la escala de medición de los datos sea ordinal. La prueba determina si las dos poblaciones son idénticas o no.

\section{Creación da la app de realidad aumentada}

Para el desarrollo de la aplicación se optó que fuera ejecutada en el sistema operativo Android de Google por ser un sistema operativo con licencia de software libre, además de ser el más utilizado en todo el mundo, con $74.45 \%$ del total del mercado, mientras que iOS registra apenas $22.85 \%$.

Se utilizó la metodología de desarrollo de software clásica denominada cascada, además de programar de forma nativa a través del ambiente de desarrollo integrado (IDE, por sus siglas en inglés) soportado por Google: Android Studio. El lenguaje de programación utilizado fue Java. 


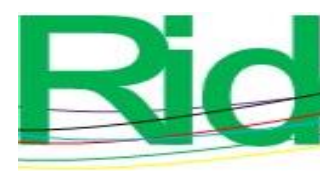

Revista Iberoamericana para la Investigación y el Desarrollo Educativo ISSN $2007-7467$

Con respecto a la tecnología seleccionada para la implementación de la RA, se analizaron diversas opciones: Vuforia, ARKit, ARCore y AR.js. Sin embargo, fue ARCore de Google la opción elegida debido a la compatibilidad que tiene con Android, con el lenguaje de programación Java y por ser de uso libre. Además, esta tecnología implementa la superposición de modelos 3D (tercera dimensión) con RA a través del reconocimiento de superficies planas, lo cual evita la necesidad de marcadores u otras opciones extra para poder utilizar la RA. Para realizar los modelos en 3D necesarios para mostrarse dentro de la aplicación, se utilizó Blender, también por ser software libre.

\section{Análisis y diseño}

Una vez que se analizaron los requerimientos, se realizó un diseño inicial de la pantalla principal donde se muestran, en forma de botones, las seis funciones básicas que se pueden trabajar (ver figura 1).

Figura 1. Pantalla inicial

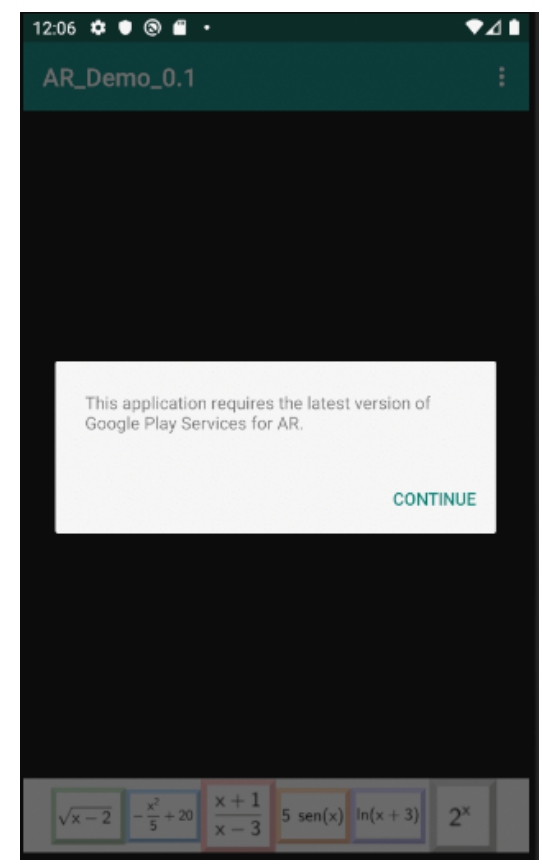

Fuente: Elaboración propia 


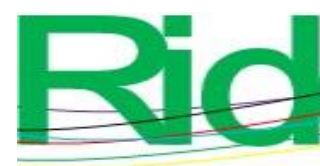

Revista Iberoamericana para la Investigación y el Desarrollo Educativo

ISSN 2007 - 7467

Figura 4. Modelo 3D de una función racional

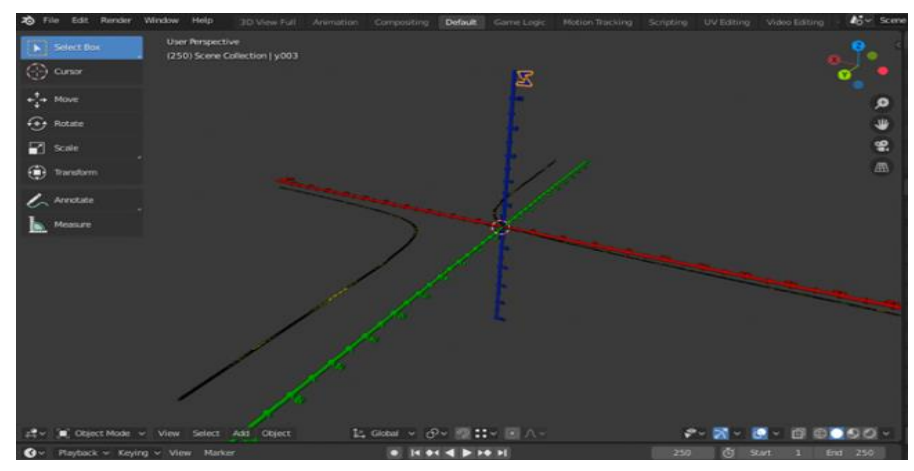

Fuente: Elaboración propia

En la figura 5 se muestra la representación tridimensional de la función trigonométrica $f(x)=5 \operatorname{sen}(x)$.

Figura 5. Modelo 3D de una función trigonométrica

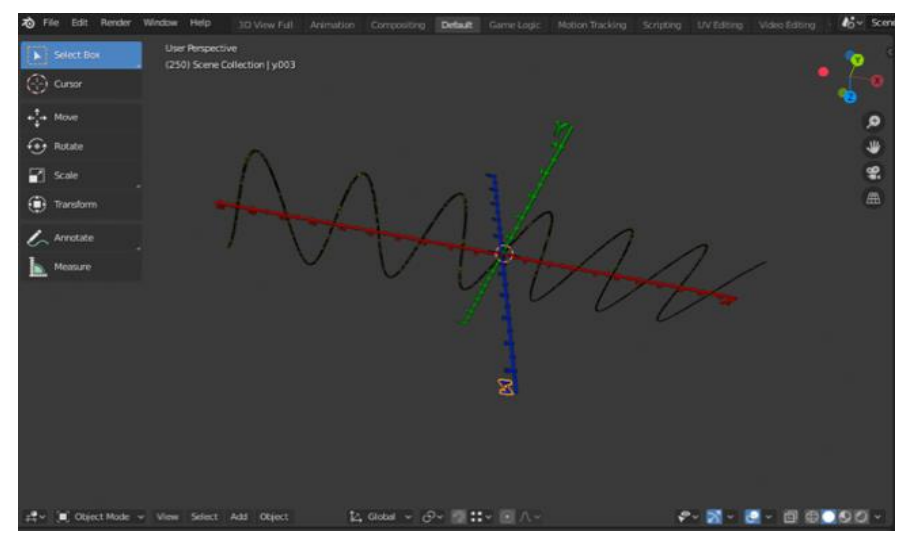

Fuente: Elaboración propia

En la figura 6 se muestra la representación tridimensional de la función exponencial $f(x)=2^{x}$ 


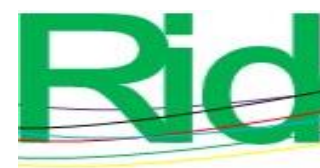

Revista Iberoamericana para la
Investigación y el Desarrollo Educativo
ISSN $2007-7467$

1) Agregando los modelos en $3 \mathrm{D}$ de las funciones a mostrar.

2) Adecuando el código necesario dentro de la aplicación.

3) Agregando el código del menú principal de opciones para mostrar la función seleccionadas.

Se obtuvo una aplicación para dispositivos móviles con sistema operativo Android que tengan una interfaz de programación de aplicaciones (API, por sus siglas en inglés) versión 24 o posterior. La funcionalidad de RA puede ser visualizada sobre una superficie plana, que será detectada automáticamente por la aplicación, y una vez identificada dicha superficie se podrá seleccionar una de las funciones que aparecen en la parte inferior del menú principal. Una aplicación real del uso de la app está en la figura 8.

Figura 8. Ejemplo real de uso de la app

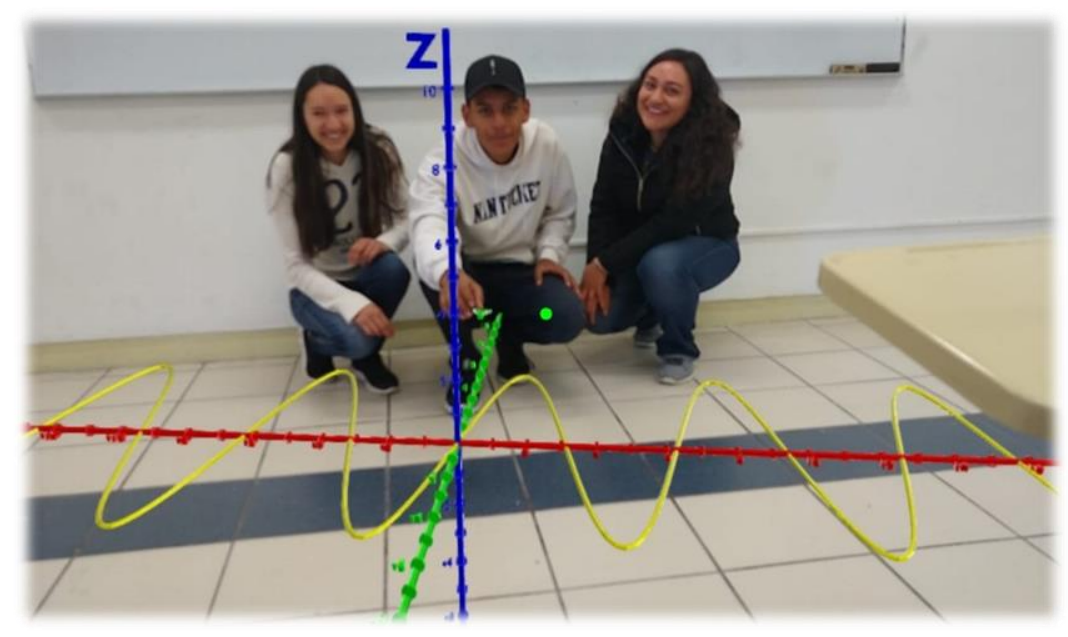

Fuente: Elaboración propia

\section{Población de estudio}

La población de este estudio fue integrada por el total de 21 alumnos que cursaron la materia de "Cálculo diferencial e integral" durante el semestre enero-junio de 2020 del ITSJ de la carrera de CP distribuidos como lo muestra la tabla 1. 
Tabla 1. Distribución de alumnos que cursaron la materia de "Cálculo diferencial e integral" durante el semestre enero-junio de 2020 del ITSJ

\begin{tabular}{|c|c|c|c|c|}
\hline Grupo & Carrera & Hombres & Mujeres & Total \\
\hline Experimental & $\mathrm{CP}$ & 4 & 7 & 11 \\
\hline Control & $\mathrm{CP}$ & 3 & 7 & 10 \\
\hline Total & & 7 & 14 & 21 \\
\hline
\end{tabular}

Fuente: Elaboración propia

\section{Recolección de datos}

En primer lugar, se recogieron dos conjuntos de calificaciones de cada grupo (control y experimental) después de aplicar el postest con un total de 36 aciertos (ver tabla 2 y 3 ). En segundo lugar, se aplicó una encuesta tomada de Martínez et al. (2017) para obtener información de los estudiantes en relación con la RA (ver anexo 1). Se verificó la confiabilidad del instrumento y se calculó un alpha de Cronbach de 0.733.

Tabla 2. Aciertos del grupo experimental

\begin{tabular}{|c|c|}
\hline Núm. & Aciertos (36) \\
\hline 1 & 34.0 \\
\hline 2 & 28.5 \\
\hline 3 & 34.0 \\
\hline 4 & 23.0 \\
\hline 5 & 24.0 \\
\hline 6 & 36.0 \\
\hline 7 & 28.0 \\
\hline 8 & 34.0 \\
\hline 9 & 17.0 \\
\hline 10 & 19.5 \\
\hline 11 & 25.5 \\
\hline
\end{tabular}

Fuente: Elaboración propia 
Tabla 3. Aciertos del grupo de control

\begin{tabular}{|c|c|}
\hline Núm. & Aciertos (36) \\
\hline 1 & 31.0 \\
\hline 2 & 15.5 \\
\hline 3 & 24.5 \\
\hline 4 & 19.5 \\
\hline 5 & 20.0 \\
\hline 6 & 25.0 \\
\hline 7 & 25.5 \\
\hline 8 & 29.5 \\
\hline 9 & 36.0 \\
\hline 10 & 27.0 \\
\hline
\end{tabular}

Fuente: Elaboración propia

\section{Análisis de datos}

Para comparar si existía o no diferencia entre las calificaciones del grupo de control y el grupo experimental, se aplicó la prueba no paramétrica de Mann-Whitney-Wilcoxon. La prueba determina si las dos poblaciones son idénticas y, por extensión, las hipótesis fueron las siguientes:

- $\quad \mathrm{H}_{0}$ : Las dos poblaciones son idénticas.

- $\mathrm{H}_{1}$ : Las dos poblaciones no son idénticas.

La prueba se utilizó para el caso de dos muestras grandes $(n \geq 10)$. Dado que los tamaños de las muestras fueron $n_{1}=11$ y $n_{2}=10$, se usó la aproximación de la distribución muestral de la suma de los rangos $T$, determinada por:

- $\quad$ Media: $\mu_{T}=(1 / 2)\left(n_{1}\right)\left(n_{1}+n_{2}+1\right)$.

- Desviación estándar: $\sigma_{T}=\sqrt{(1 / 12)\left(n_{1}\right)\left(n_{2}\right)\left(n_{1}+n_{2}+1\right)}$.

- $\quad$ Valor estadístico de prueba: $Z=\left(T-\mu_{T}\right) / \sigma_{T}$ donde $T$ es la suma de los rangos.

- $\quad$ Se consideró un nivel de significancia $\alpha=0.05$.

Además de realizar los cálculos de las fórmulas anteriores de forma manual, se utilizó el software SPSS versión 22 para verificarlos. 
Los resultados fueron:

$$
\begin{array}{ll}
\text { - } & \mu_{T}=121 \\
\text { - } & \sigma_{T}=14.201 \\
\text { - } & T=130.5 \\
\text { - } & Z=0.67
\end{array}
$$

En la figura 9 se representan las cantidades anteriores; además, se observa que el valor de $Z$ se encuentra en el área donde $\mathrm{H}_{0}$ no se rechaza, es decir, como no tenemos evidencia para rechazar $\mathrm{H}_{0}$ podemos concluir que las dos poblaciones son idénticas.

Figura 9. Distribución normal

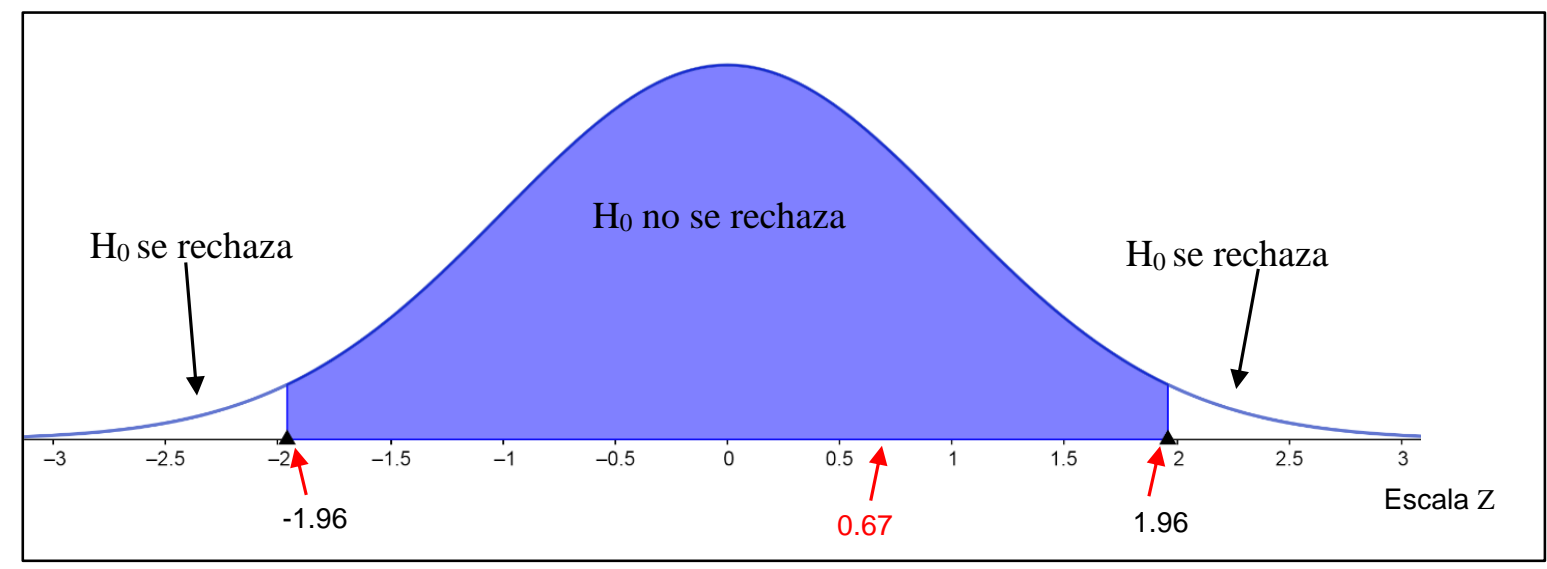

Fuente: Elaboración propia

\section{Resultados}

El análisis de los resultados de la investigación se realizó en dos apartados: la prueba no paramétrica de Mann-Whitney-Wilcoxon y los resultados del anexo 1.

Como se observa en la figura $9, \mathrm{H}_{0}$ no se rechaza, es decir, como no tenemos evidencia para rechazar $\mathrm{H}_{0}$ podemos concluir que las dos poblaciones son idénticas. Esto significa que en nuestro estudio de caso el uso de la RA no mejora el rendimiento académico de los estudiantes en el tema de funciones de la asignatura de Cálculo. Las calificaciones obtenidas en el postest del grupo de control y del grupo experimental son similares. Por otro lado, veamos los resultados obtenidos en el anexo 1, del que se recogió información de los estudiantes en relación con su conocimiento previo de la RA, la disponibilidad de recursos para su aplicación, criterios motivacionales y criterios sobre la el uso de la RA. 


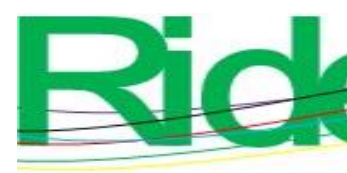

Revista Iberoamericana para la Investigación y el Desarrollo Educativo ISSN $2007-7467$

Se tienen algunos datos tales como los siguientes: $36.4 \%$ son hombres y $63.6 \%$ son mujeres, una edad promedio de 18.3 y una desviación estándar de 0.47 . En cuanto a las preguntas, $81.8 \%$ dice que conocía el término $R A$, aunque $54.5 \%$ no había empleado la RA; $81.8 \%$ menciona que el empleo de la RA le ayudó a mantener la atención en el transcurso de la asignatura; $72.7 \%$ considera que la RA no es tan compleja; $81.8 \%$ dice que la interacción con la RA le ayudó en la interacción con la asignatura; 81.8 \% consideró que la información que brinda la RA despertó o aumentó el interés por la asignatura, y $54.5 \%$ evaluí que la cantidad de actividades fueron muy pocas. Finalmente, $90.9 \%$ consideró que en la medida que trabajó con la RA se sintió más seguro de que podía aprender con esta herramienta.

\section{Discusión}

La RA es una herramienta que está siendo muy utilizada en diferentes entornos. Introducirla en el proceso de enseñanza-aprendizaje del cálculo, de acuerdo con los resultados obtenidos, no tiene un impacto significativo. No obstante, en el cuestionario aplicado la mayoría de los estudiantes consideró que se sentía más segura al aprender con esta herramienta, lo que coincide con el estudio cuasiexperimental realizado a 63 estudiantes en la Escuela Superior Politécnica de Chimborazo del Ecuador, cuyos resultados muestran que la RA fortalece escenarios formativos más motivadores, colaborativos e interactivos (Barba et al., 2015).

Por otra parte, Redondo et al. (2017) realizaron un experimento similar con 25 estudiantes usando la RA en dispositivos móviles y encontraron que hubo una mejora significativa en las calificaciones del grupo experimental; así, en este caso en específico, demostraron que la tecnología de RA es una herramienta valiosa en el campo de la arquitectura. Dicho estudio evidencia que, efectivamente, el uso de la RA mejora las calificaciones finales de los estudiantes, sin embargo, la comparación de ambos estudios se realiza con respecto al tamaño de la muestra, es decir, son muestras pequeñas (similares).

En este mismo orden de ideas, Parroquín et al. (2013) describen el desarrollo de una aplicación de software que usa la tecnología de RA para la enseñanza de la física; aquí también se experimentó una práctica desarrollada con RA en un grupo experimental, y en otro grupo control se desarrolló la práctica en el modo habitual, sin uso del software. En una parte de sus conclusiones aluden a que es importante la realización de más pruebas de 


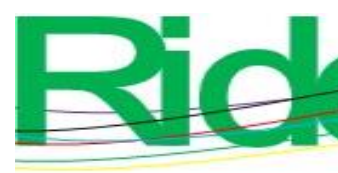

Revista Iberoamericana para la Investigación y el Desarrollo Educativo ISSN $2007-7467$

aprendizaje. La comparación con este estudio se exhibe debido a que la app fue creada únicamente para el uso en el experimento en el área de matemáticas.

Es un hecho que, con el avance de la tecnología y con el uso que esta ha tenido debido a la pandemia por la covid-19, la práctica docente tendrá que buscar y construir herramientas que sean adaptables a diversas situaciones, herramientas que en todo momento contribuyan a que los estudiantes en general, y en particular los de matemáticas, se apropien del conocimiento desde una nueva perspectiva, desde su visión, la cual ya no se puede ni debe separar de la tecnología.

\section{Conclusiones}

Las aplicaciones de RA son cada vez más utilizadas en nuestro entorno: medicina, publicidad, ingeniería, turismo, museos, juegos, etc., y dentro del sector educativo esta tecnología también está presente. Esto en gran parte debido al uso de teléfonos inteligentes y tabletas. En el experimento realizado se tenía como pregunta: ¿el uso de la RA permitirá mejorar el rendimiento académico de los alumnos en el tema de funciones de la asignatura de Cálculo? El estudio de caso nos muestra que no hay diferencia si se utiliza RA como herramienta de apoyo o se presentan las clases de forma tradicional, es decir, en cuanto a calificaciones no hay diferencia significativa. Sin embargo, los estudiantes que utilizaron la RA consideran que esta les ayudó a mantener la atención en el transcurso de la asignatura; asimismo, despertó o aumentó el interés por la asignatura, y, además, un alto porcentaje consideró que en la medida que trabajó con la RA se sintió más seguro de que podía aprender.

Teniendo en cuenta lo anterior, se puede considerar que la RA posee un alto potencial para ser utilizada en entornos educativos y que, además, la RA despierta interés entre los estudiantes. Una desventaja de este estudio es el tamaño de la muestra; será necesario para los siguientes estudios aumentar el tamaño de esta y, por otro lado, crear apps para temas diferentes. Si bien es cierto que la RA no será la solución perfecta para las necesidades de las aplicaciones relacionadas con la educación, es una opción que no se puede dejar de considerar. 


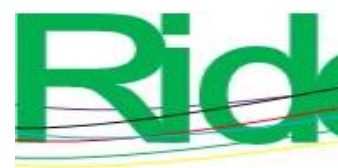

Revista Iberoamericana para la
Investigación y el Desarrollo Educativo
ISSN $2007-7467$

\section{Futura líneas de investigación}

El empleo de la tecnología que puede complementar la educación tradicional y potenciar el conocimiento constituye un desafío para los docentes, sobre todo en el caso de la RA. Será un reto constante para los profesores la innovación de sus actividades de enseñanza dentro de las aulas de clase. Para el desarrollo de futuras investigaciones se proponen las siguientes acciones: respecto al software, se pueden perfeccionar algunos aspectos de la app de RA, existen oportunidades de crear una más dinámica, esto es, que el estudiante tenga la opción de agregar su propia función y que esta se visualice en RA, ya que la creada en esta investigación era estática y solo estaba programada para usarse con seis funciones diferentes.

Considerando que la RA permite visualizar el problema, se propone replicar el estudio en otros temas de matemáticas que son visuales, tales como la pendiente de la recta tangente, integral definida, transformaciones lineales, sistema de ecuaciones lineales y operaciones con vectores, por mencionar algunos. Finalmente, si bien es cierto que no se encontró diferencia significativa en el rendimiento académico con o sin el uso de la RA, puede repetirse el experimento con muestras más grandes, además de incorporar profesores no especialistas en programación de otras asignaturas para la creación de objetos con RA.

\section{Agradecimientos}

Para la realización de este trabajo participaron 21 estudiantes de la carrera de CP del ITSJ, a quienes se les reconoce su apoyo para el desarrollo del experimento. También agradecemos a la alumna Brenda Botello Trujillo, estudiante del ITSJ, quien apoyó en la realización de este proyecto. Igualmente reconocemos a A. A. English, Alonso García, por su colaboración en la traducción y revisión técnica. Y por supuesto, al ITSJ por el apoyo brindado para la culminación de esta investigación. 


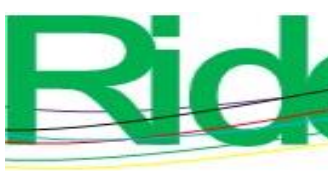

Revista Iberoamericana para la
Investigación y el Desarrollo Educativo ISSN $2007-7467$

\section{Referencias}

Álvarez, A., Castillo, M., Pizarro, J. y Espinoza, E. (2017). Realidad aumentada como apoyo a la formación de ingenieros industriales. Formación Universitaria, 10(2), 31-42.

Amadeo, R. (29 de agosto de 2017). Google's ARCore brings augmented reality to millions of Android devices. Ars Technica. Recuperado de: https://arstechnica.com/gadgets/2017/08/googles-arcore-brings-augmented-realityto-millions-of-android-devices/

Anderson, D., Sweeney, D. y Williams, T. (2008). Estadística para la administración y economía (10. ${ }^{\mathrm{a}}$ ed.). México: Cengage Learning.

Azuma, R. T. (1997). A Survey of Augmented Reality. Presence-Teleoperators and Virtual Environments, 6 (4), 355-385.

Ballesteros, J. A. y Bernal, L. (2016). Metodología para la construcción de objetos virtuales de aprendizaje, apoyada en realidad aumentada. Sophia, 13(1), 4-12. Recuperado de http://dx.doi.org/10.18634/sophiaj.13v.1i.209.

Barba, R. G., Yasaca, S. y Manosalvas, C. A. (2015). Impacto de la realidad aumentada móvil en el proceso enseñanza-aprendizaje de estudiantes universitarios del área de medicina. Investigar con y para la Sociedad, 3, 1421-1429.

Buitrago, R. D. (2015). Incidencia de la realidad aumentada sobre el estilo cognitivo: caso para el estudio de las matemáticas. Educación y Educadores, 18(1), 27-41.

Cabero, J. and Barroso, J. M. (2016). The Educational Possibilities of Augmented Reality. Journal of New Approaches in Educational Research, 5(1), 44-50. Retrieved from https://files.eric.ed.gov/fulltext/EJ1127264.pdf.

Cabero, J., Fernández, B. y Marín, V. (2017). Dispositivos móviles y realidad aumentada en el aprendizaje del alumnado universitario. RIED. Revista Iberoamericana de Educación a Distancia, 20(2), 167-185. Recuperado de http://dx.doi.org/10.5944/ried.20.2.17245.

Cupitra, A. y Duque, E. T. (2018). Profesores aumentados en el contexto de la realidad aumentada: una reflexión sobre su uso pedagógico. El Ágora USB, 18(1), 244-254. Recuperado de http://dx.doi.org/10.21500/16578031.3178.

De la Horra, I. (2017). Realidad aumentada, una revolución educativa. Edmetic, 6(1), 9-22. 
Del Cerro, F. y Morales, G. (2017). Realidad Aumentada como herramienta de mejora de la inteligencia espacial en estudiantes de educación secundaria. RED. Revista de Educación a Distancia, (54), 1-14. Recuperado de http://dx.doi.org/10.6018/red/54/5.

Edel, R. (2003). El rendimiento académico: concepto, investigación y desarrollo. REICE. Revista Electrónica Iberoamericana sobre Calidad, Eficacia y Cambio en Educación, $1(2), 1-15$.

Lopes, L. M., Sartor, K. N., Pozzebon, E. and Ferenhof, H., (2019). Inovações educacionais com o uso da realidade aumentada: uma revisão sistemática. EDUR. Educação em Revista, 35. Recuperado de http://dx.doi.org/10.1590/0102-4698197403.

Lorenzo, G. y Scagliarini, C. (2018). Revisión bibliométrica sobre la realidad aumentada en Educación. Revista General de Información y Documentación, 28(1), 45-60. doi: https://doi.org/10.5209/RGID.60805

Maquilón, J., Mirete, A. y Avilés, M. (2017). La realidad aumentada (RA). Recursos y propuestas para la innovación educativa. Revista Electrónica Interuniversitaria de Formación del Profesorado, 20 (2), 183-203.

Martínez, H., García, A. y Escalona, J. C. (2017). Modelos de realidad aumentada aplicados a la enseñanza de la química en el nivel universitario. Revista Cubana de Química, 29(1), 13-25.

Ocampo, J., Martínez, Á., De las Fuentes, M. y Zataraín, J. (2010). Reprobación y deserción en la Facultad de Ingeniería Mexicali de la Universidad Autónoma de Baja California. Ponencia.

Parroquín, P., Ramírez, J., González, V. y Mendoza, A. (2013). Aplicación de la realidad aumentada en la enseñanza de la física. Cultura Científica y Tecnológica, (51), 182192.

Pedraza, C., Amado, O., Lasso, E. y Munévar, P. (2017). La experiencia de la realidad aumentada (RA) en la formación del profesorado en la Universidad Nacional Abierta y a Distancia (UNAD) Colombia. Pixel-Bit. Revista de Medios y Educación, (51), 111-131.

Purcell, E., Varberg, D. y Rigdon, S. (2007). Cálculo diferencial e integral (9. ${ }^{\mathrm{a}}$ ed.). México: Prentice Hall.

Redondo, E., Fonseca, D., Sánchez, A. y Navarro, I. (2017). Formación de urbanistas usando realidad aumentada y tecnologías de aprendizaje móvil. RIED. Revista 


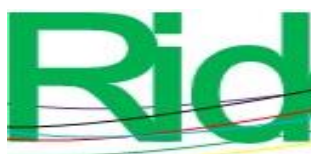

Revista Iberoamericana para la
Investigación y el Desarrollo Educativo
ISSN $2007-7467$

Iberoamericana de Educación a Distancia, 20(2), 141-165. Recuperado de http://dx.doi.org/10.5944/ried.20.2.17675.

Rubio, S., Cabero Almenara, J., Leiva Olivencia, J. J., Moreno Martínez, N. M., Barroso Osuna, J. y López Meneses, E. (2016). Realidad aumentada y educación. Barcelona: Ediciones Octaedro. Pixel-Bit. Revista de Medios y Educación, (50), 237-238.

Saltan, F. and Arslan, Ö. (2017). The Use of Augmented Reality in Formal Education: A Scoping Review. Eurasia Journal of Mathematics, Science \& Technology Education, 13(2), 503-520. Retrieved from https://www.ejmste.com/article/the-use-ofaugmented-reality-in-formal-education-a-scoping-review-4676.

Tejedor, F. J. T., \& Muñoz-Repiso, A. G. V. (2007). Causas del bajo rendimiento de estudiante universitario (en opinión de los profesores y alumnos): propuestas de mejora en el marco del EEES. Revista de Educación, (342), 419-442. 


\begin{tabular}{|c|c|}
\hline & $\begin{array}{l}\text { Revista lberoamericana para la } \\
\text { Investigación y el Desarrollo Educativo } \\
\text { ISSN } 2007-7467\end{array}$ \\
\hline Rol de Contribución & Autor (es) \\
\hline Conceptualización & $\begin{array}{l}\text { Efrén Berumen López (Igual) Salvador Acevedo Sandoval } \\
\text { (Igual) }\end{array}$ \\
\hline Metodología & Efrén Berumen López \\
\hline Software & Salvador Acevedo Sandoval \\
\hline Validación & Efrén Berumen López (Igual) Susana Reveles Gamboa (Igual) \\
\hline Análisis Formal & $\begin{array}{l}\text { Efrén Berumen López (Igual) Salvador Acevedo Sandoval } \\
\text { (Igual) }\end{array}$ \\
\hline Investigación & $\begin{array}{l}\text { Efrén Berumen López (Principal) Susana Reveles Gamboa } \\
\text { (que apoya) }\end{array}$ \\
\hline Recursos & $\begin{array}{l}\text { Efrén Berumen López (Igual) Salvador Acevedo Sandoval } \\
\text { (Igual) Susana Reveles Gamboa (Igual) }\end{array}$ \\
\hline Curación de datos & $\begin{array}{l}\text { Efrén Berumen López (Igual) Salvador Acevedo Sandoval } \\
\text { (Igual) }\end{array}$ \\
\hline $\begin{array}{l}\text { Escritura - Preparación del } \\
\text { borrador original }\end{array}$ & $\begin{array}{l}\text { Efrén Berumen López (Principal) Salvador Acevedo Sandoval } \\
\text { (Igual) Susana Reveles Gamboa (Igual) }\end{array}$ \\
\hline $\begin{array}{l}\text { Escritura - Revisión y } \\
\text { edición }\end{array}$ & $\begin{array}{l}\text { Efrén Berumen López (Principal) Salvador Acevedo Sandoval } \\
\text { (Igual) Susana Reveles Gamboa (Igual) }\end{array}$ \\
\hline Visualización & $\begin{array}{l}\text { Efrén Berumen López (Principal) Salvador Acevedo Sandoval } \\
\text { (Igual) Susana Reveles Gamboa (Igual) }\end{array}$ \\
\hline Supervisión & Efrén Berumen López \\
\hline Administración de Proyectos & Efrén Berumen López \\
\hline Adquisición de fondos & $\begin{array}{l}\text { Efrén Berumen López (Igual) Salvador Acevedo Sandoval } \\
\text { (Igual) Susana Reveles Gamboa (Igual) }\end{array}$ \\
\hline
\end{tabular}




\section{Anexo 1. Encuesta de satisfacción}

Estamos realizando una investigación sobre el uso de una app de realidad aumentada en la educación superior, te pedimos que respondas a todas las preguntas con la mayor sinceridad. Tus respuestas serán tratadas de forma confidencial. Al lado de cada afirmación se presentan cinco opciones, en una escala de uno a cinco. Lee cada frase detenidamente y a continuación rodea el número que mejor se relacione con lo que tú haces o piensas. Debes escoger solo uno.

- $\quad l=$ Totalmente en desacuerdo.

- $\quad 2=$ No totalmente de acuerdo.

- $3=$ De acuerdo pero...

- 4 = De acuerdo.

- $5=$ Totalmente de acuerdo.

¡Gracias por tu colaboración!

Tabla 4. Encuesta de Satisfacción

\begin{tabular}{|c|c|c|c|c|c|}
\hline Indica tu género & \multicolumn{2}{|l|}{ Hombre } & \multicolumn{3}{|l|}{ Mujer } \\
\hline \multicolumn{6}{|l|}{ Escribe tu edad actual (años) } \\
\hline Indica la carrera que estudias & ISC & IM & IIA & LA & $\mathrm{CP}$ \\
\hline Estudios de tu papá & Ninguno & Primaria & Secundaria & Bachillerato & Superior \\
\hline Estudios de tu mamá & Ninguno & Primaria & Secundaria & Bachillerato & Superior \\
\hline Pregunta & 1 & 2 & 3 & 4 & 5 \\
\hline $\begin{array}{l}\text { 1) Conozco el término realidad } \\
\text { aumentada. }\end{array}$ & 1 & 2 & 3 & 4 & 5 \\
\hline $\begin{array}{l}\text { 2) He empleado la realidad aumentada } \\
\text { antes de este curso. }\end{array}$ & 1 & 2 & 3 & 4 & 5 \\
\hline $\begin{array}{l}\text { 3) Cuento con los recursos necesarios } \\
\text { para el uso de la realidad aumentada } \\
\text { en el estudio independiente. }\end{array}$ & 1 & 2 & 3 & 4 & 5 \\
\hline $\begin{array}{l}\text { 4) El empleo de la realidad aumentada } \\
\text { en las clases me ayudó a mantener la }\end{array}$ & 1 & 2 & 3 & 4 & 5 \\
\hline
\end{tabular}




\begin{tabular}{|l|c|c|c|c|c|}
\hline $\begin{array}{l}\text { atención en el transcurso de la } \\
\text { asignatura. }\end{array}$ & 1 & 2 & 3 & 4 & 5 \\
\hline $\begin{array}{l}\text { 5) La realidad aumentada es tan } \\
\text { compleja que me fue difícil emplearla. }\end{array}$ & 1 & 2 & 3 & 4 & 5 \\
\hline $\begin{array}{l}\text { 6) La interacción con la realidad } \\
\text { aumentada me ayudó en la interacción } \\
\text { con la asignatura. }\end{array}$ & 1 & 2 & 3 & 4 & 5 \\
\hline $\begin{array}{l}\text { 7) La información que brinda la } \\
\text { realidad aumentada me despertó o } \\
\text { aumentó mi interés por la asignatura. }\end{array}$ & 1 & 2 & 3 & 4 & 5 \\
\hline $\begin{array}{l}\text { 8) La cantidad de actividades en las } \\
\text { que se empleó la realidad aumentada } \\
\text { fueron muy pocas. }\end{array}$ & 1 & 2 & 3 & 4 & 5 \\
\hline $\begin{array}{l}\text { 9) La variedad de temas presentados } \\
\text { en realidad aumentada fue amplia. }\end{array}$ & 1 & 2 & 3 & 4 & 5 \\
\hline $\begin{array}{l}\text { 10) En la medida que trabajé con la } \\
\text { Realidad Aumentada me sentí más } \\
\text { seguro de que podía aprender con esta } \\
\text { herramienta. }\end{array}$ & 1 & 2 & 3 & 4 & 5 \\
\hline $\begin{array}{l}\text { 11) Los modelos presentados en la } \\
\text { realidad aumentada fueron muy pocos. }\end{array}$ & 1 & & & & \\
\hline
\end{tabular}

Fuente: Tomada de Martínez et al. (2017). 